\title{
Análisis de los cambios en la auto-eficacia lectora a nivel evolutivo en la Educación Secundaria Obligatoria
}

\author{
Fátima Olivares, Raquel Fidalgo y Olga Arias-Gundín \\ Universidad de León (España)
}

\begin{abstract}
Se analizan los cambios en el nivel de auto-eficacia lectora del alumnado de Educación Secundaria Obligatoria desde un punto de vista evolutivo. La muestra la formaron 492 alumnos/as de $1^{\circ}$ a $4^{\circ}$ de la ESO (de 12 a 16 años), pertenecientes a cuatro centros públicos y cuatro centros privados-concertados de la provincia de León (283 hombres y 209 mujeres). Tras la aplicación del Cuestionario de Auto-eficacia hacia la lectura y los correspondientes análisis, se concluye la existencia de diferencias estadísticamente significativas en el patrón evolutivo del nivel de auto-eficacia lectora entre los cursos de $1^{\circ}$ a $4^{\circ}$ de Educación Secundaria Obligatoria en función de la edad, en relación a las medidas de auto-eficacia total y auto-eficacia en relación a los procesos de decodificación y fluidez, mostrándose un aumento del nivel de auto-eficacia lectora a medida que aumenta la edad. Sin embargo, no se observan cambios en relación a la auto-eficacia en los procesos relacionados con la formación del modelo de situación y modelo textual. Se discuten las implicaciones educativas, así como las limitaciones y perspectivas futuras de investigación.
\end{abstract}

Palabras clave: Auto-eficacia, comprensión lectora, motivación lectora, desarrollo, secundaria.

Analysis of Developmental Changes on Reading Self-efficacy through Compulsory Secondary Education. This study analyzes changes on students 'reading self-efficacy beliefs through the compulsory Secondary Education from a developmental perspective. Sample comprised 492 students from $1^{\text {st }}$ to $4^{\text {th }}$ Secondary Grades (from 12 to 16 years old), belonged to four public schools and four semi-private schools of León (283 males and 209 females). After the application of the Reading Self-efficacy Scale and the subsequent analysis, results suggested statistically significant differences in the developmental pattern of reading self-efficacy across the $1^{\text {st }}$ to $4^{\text {th }}$ grades of compulsory Secondary Education, specifically, in the global reading self-efficacy and the subscale of decoding and fluency reading self-efficacy. They showed an increase in reading selfefficacy along the grades. However, we did not find changes in the other reading selfefficacy subscales, reading self-efficacy in relation to the construction of the text model and the situation model of reading. Educational implications, limitations and future lines of research of this study are discussed.

Key words: Self-efficacy, reading comprehension, reading motivation, development, secondary education.

Correspondencia: Raquel Fidalgo. Área de Psicología Evolutiva y de la Educación. Universidad de León. Facultad de Educación. Campus de Vegazana, s/n. C.P. 24071. León (España). E-mail: rfidr@unileon.es 
Investigaciones recientes en el campo de la comprensión lectora resaltan cómo los factores cognitivos no son suficientes para explicar el nivel de comprensión lectora de los estudiantes. La lectura requiere un esfuerzo que los estudiantes eligen hacer o no hacer, elección que en gran parte está determinada por su nivel de motivación, lo que convierte a la lectura en una actividad motivada (Guthrie y Wigfield, 2000); lo que nos lleva a considerar junto a los factores cognitivos el papel clave de los factores motivacionales en la comprensión lectora. De entre ellos, un aspecto que parece tener especial importancia como consecuencia de la influencia que ejerce sobre la motivación, son las creencias de auto-eficacia percibida (Pajares, 2003; Zinmerman, 2000). Surge así, la auto-eficacia, como alto predictor del nivel de comprensión lectora de los estudiantes (Linnenbrink y Pintrich, 2003).

Siguiendo a Bandura (1997), definimos la auto-eficacia lectora como el juicio o auto-evaluación individual para realizar con éxito una tarea, en este caso la actividad lectora; influyendo de modo directo en el dominio y adquisición de la habilidad lectora a través de tres niveles de compromiso: conductual, cognitivo y motivacional. En este punto, diversas investigaciones han concluido como una alta auto-eficacia aumenta el compromiso, motivación y por lo tanto, el uso de estrategias de comprensión lectora (Wigfield, Guthrie, Tonks y Perencevich, 2004), indicando cómo los sujetos con una alta auto-eficacia tienden a estar más motivados hacia las tareas, lo que repercute en una mayor implicación, persistencia y esfuerzo en su aprendizaje, y por lo tanto en un mayor éxito o rendimiento del alumno en su aprendizaje.

La auto-eficacia también es clave para un aprendizaje auto-regulado (Pajares, 2003), de modo que el alumno adquiere mayor autocontrol y autoconocimiento de los procesos implicados en la comprensión lectora. Los lectores pueden aprender estrategias cognitivas y metacognitivas, sin embargo, su uso está relacionado con las creencias de auto-eficacia en el proceso lector. Cuando la auto-eficacia y creencias motivacionales son altas para la comprensión del texto, los alumnos utilizan estrategias lectoras, que mantienen y generalizan (Nelson y Manset-Williamson, 2006).

Dada por tanto, la importancia de la auto-eficacia en la comprensión lectora, en los últimos años su estudio ha pasado a ser el foco de distintas investigaciones a nivel internacional, sin embargo, tal como muestran revisiones empíricas en este campo (ver Olivares y Fidalgo, 2012), desde un punto de vista evolutivo, no se muestra una conclusión o tendencia clara en relación a los cambios en el nivel de auto-eficacia lectora a través de los cursos escolares. Mientras que diversas investigaciones han concluido un declive en las creencias de competencia de los estudiantes durante la adolescencia (Kelley y Decker, 2009; Lau, 2009a; 2009b), que podría ser explicado por una parte, porque los alumnos de cursos inferiores tienden a sobreestimar sus capacidades y a medida que crecen, realizan juicios más realistas de sus habilidades, o porque las tareas de lectura a las que se enfrentan en las etapas superiores suponen un nivel de exigencia y 
de esfuerzo mayor, lo que puede ir en detrimento de su nivel de competencia percibida. Sin embargo, otras investigaciones informan de lo contrario a lo obtenido en los estudios anteriores. Así, Mucherah y Yoder (2008) concluyen que los estudiantes de los cursos superiores presentan un mayor nivel de auto-eficacia lectora. Resultados que podrían ser explicados como consecuencia del proceso de adquisición de la competencia lectora, puesto que los estudiantes de cursos superiores tienen un mayor nivel de competencia lectora que los de cursos inferiores, lo que debería ir unido a un alto nivel de auto-eficacia lectora.

La falta de acuerdo en el ámbito de la auto-eficacia lectora, mostrando resultados contradictorios en relación al nivel de auto-eficacia en función de la edad, justifican la necesidad de un estudio como el que aquí se presenta, con el objetivo de analizar el nivel de auto-eficacia lectora de los alumnos desde un punto de vista evolutivo. Para ello, se compararon cuatro cohortes de edad correspondientes a los cuatro cursos de la Educación Secundaria Obligatoria; precisamente por ser este periodo educativo en el que las diferentes evaluaciones e informes comparativos del rendimiento lector a nivel internacional han puesto de manifiesto las dificultades y bajo rendimiento generalizado que presentan nuestros alumnos, significativamente menor que el de otros países occidentales con sistemas educativos similares al nuestro (OCDE, 2009). Es por ello esta etapa un periodo crítico en la comprensión lectora, de ahí que tome un especial interés también a nivel práctico el detectar periodos críticos en el desarrollo de la autoeficacia lectora en estas edades, que precisamente podrían estar incidiendo negativamente en el rendimiento lector del alumnado de la Etapa de Educación Secundaria Obligatoria. Aspecto de gran relevancia a nivel educativo o aplicado, puesto que facilitará la posibilidad de dar una respuesta instruccional ajustada a dichas necesidades, consiguiendo con ello la finalidad última de mejorar la competencia lectora de los estudiantes.

\section{MÉTODO}

\section{Participantes}

Se seleccionó una muestra amplia y representativa de alumnos de la provincia de León, con un total de 492 alumnos de $1^{\circ}$ a $4^{\circ}$ de Educación Secundaria (12 a 16 años), escolarizados en cuatro centros públicos y cuatro centros privados-concertados. De los cuales atendiendo al género de los mismos, 283 fueron hombres y 209 mujeres. En la tabla 1 se recoge la distribución de la muestra por curso y género.

En la selección de la muestra se controló que respondiera a características generales de la población, asegurando la representatividad de la población a la que se quieren generalizar los resultados. Se excluyeron de la muestra aquellos alumnos 
diagnosticados con algún tipo de necesidades educativas específicas de apoyo educativo. La distribución de la muestra fue equivalente por género.

Tabla 1. Distribución de la Muestra en Función del Género y Curso

\begin{tabular}{llccccc}
\hline & & \multicolumn{3}{c}{ Curso } & \\
\cline { 2 - 7 } & & $1^{\circ}$ ESO & $2^{\circ}$ ESO & $3^{\circ}$ ESO & $4^{\circ}$ ESO & Total género \\
\hline \multirow{2}{*}{ Género } & Hombres & 78 & 79 & 69 & 57 & 283 \\
\cline { 2 - 7 } & Mujeres & 79 & 84 & 52 & 64 & 209 \\
\hline Total Curso & & 157 & 163 & 121 & 121 & 492 \\
\hline Edad mín-máx & $12-13$ años & $13-14$ años & $14-15$ años & $15-16$ años & \\
\hline
\end{tabular}

\section{Medidas}

Auto-eficacia lectora. Para la evaluación de la auto-eficacia se utilizó la Escala de auto-eficacia hacia la lectura (Fidalgo, Arias-Gundín y Olivares, 2013). Escala fundamentada en los actuales modelos teóricos de la lectura (Kintsch y Rawson, 2005), y diseñada en base a la actual guía de Bandura para la evaluación de la auto-eficacia (Bandura, 2006).

Está formada por 15 ítems, distribuidos en función de la auto-eficacia en relación con los procesos de decodificación y fluidez, en relación a los procesos implicados en la formación del texto base y en relación a los procesos relacionados con la formación del modelo de situación. De los 15 ítems, 9 pertenecen a la escala de autoeficacia para la comprensión lectora de Mattus y colaboradores desarrollada en base al modelo teórico de mediación directa e inferencial, y validada experimentalmente de forma preliminar (Mattus, Pérez, Pujol y Casasola, 2011); y adicionalmente se añadieron otros 6 ítems. Estos se distribuyen en las siguientes sub-escalas de auto-eficacia: relacionada con los procesos de decodificación y fluidez, con los procesos y factores implicados en la formación del texto base y con la formación del modelo de situación. En cada uno de los ítems se pregunta al alumnado en qué medida cree que puede lograr en la siguiente tarea de lectura y resumen de un texto cada una de las acciones que se describen a continuación. Para su respuesta, el alumnado puede utilizar cualquier número dentro de la escala de 0-100, en la que el 0 significa estar muy seguro de no poder hacerlo, y el 100 estar muy seguro de poder hacerlo.

En cuanto a la fiabilidad del cuestionario por consistencia interna, se muestra una alta fiabilidad con un $\alpha$ de Cronbach .89 para la escala completa.

Tarea de Lectura y Resumen. Dado que la auto-eficacia lectora es dependiente de la tarea, se utilizó para la evaluación de la comprensión lectora, la tarea de lectura y resumen de dos textos expositivos tomados del Test de estrategias de comprensión lectora, TEC (Vidal-Abarca, Gilabert, Martínez, Sellés, Abad y Ferrer, 2007). Las temáticas de los textos, los sioux con una lontigud de 548 palabras, y los pingüinos con una longitud de 469 palabras, fueron distribuidas de forma contrabalanceada por género y curso. Los alumnos tenían que leer y hacer un resumen con el texto delante y sin límite 
de tiempo. No se les dio ninguna instrucción específica, simplemente debían leer el texto intentando comprenderlo muy bien y hacer un resumen que recogiera las ideas principales del texto.

\section{Procedimiento}

Tras la revisión a nivel empírico y teórico en relación a la auto-eficacia lectora, se procedió al contacto con los centros educativos (públicos y concertados) de León. Una vez obtenidas las correspondientes autorizaciones se procedió a la evaluación de la auto-eficacia y la comprensión lectora. La evaluación fue realizada por personal especializado, en diferentes sesiones y bajo las mismas condiciones de aplicación. Tras la codificación de los datos se realizaron los análisis multivariado de la varianza manovas tomando como variables dependientes todas las medidas de auto-eficacia, la media de auto-eficacia total, y la auto-eficacia en relación a la construcción del modelo de situación, del modelo de texto base y en relación a los procesos de bajo nivel de la lectura procesos de decodificación y fluidez, y como variable independiente el curso escolar.

\section{RESULTADOS}

\section{Cuestionario de Auto-eficacia}

Los resultados obtenidos del análisis quedan reflejados en la tabla 2 en la que se muestran los estadísticos descriptivos por grupo. Se encontraron diferencias estadísticamente significativas en la autoeficacia lectora entre los cursos, Lambda de Wilks $=.966 ; F_{(9,1353.31)}$ y $p=.023$, obteniéndose diferencias estadísticamente significativas en la medida de auto-eficacia en relación a los procesos de decodificación y fluidez ( $p=.012)$, y próximas a la significatividad en la medida de auto-eficacia total $(p=.051)$. No presentando diferencias en el nivel de auto-eficacia lectora en función del curso en relación a la construcción del modelo de situación ( $p=.111)$ y modelo textual $(p=.88)$.

Tabla 2. Diferencias Estadísticamente Significativas en el Nivel de Auto-eficacia Lectora desde un Punto de Vista Evolutivo en Alumnos de Educación Secundaria Obligatoria

\begin{tabular}{|c|c|c|c|c|c|c|c|c|c|c|}
\hline \multirow[t]{2}{*}{ Variables } & \multicolumn{2}{|c|}{$\begin{array}{l}1^{\circ} \mathrm{ESO} \\
N=157\end{array}$} & \multicolumn{2}{|c|}{$\begin{array}{l}2^{\circ} \mathrm{ESO} \\
N=163\end{array}$} & \multicolumn{2}{|c|}{$\begin{array}{l}3^{\circ} \mathrm{ESO} \\
N=121\end{array}$} & \multicolumn{2}{|c|}{$\begin{array}{l}4^{\circ} \mathrm{ESO} \\
N=121\end{array}$} & \multirow[t]{2}{*}{$F$} & \multirow[t]{2}{*}{$p$} \\
\hline & $M$ & $D t$ & $M$ & $D t$ & $M$ & $D t$ & $M$ & $D t$ & & \\
\hline Auto-eficacia total & 71.89 & 15.21 & 70.85 & 13.40 & 74.96 & 13.32 & 74.12 & 13.78 & 2.60 & .051 \\
\hline Auto-eficacia modelo situación & 72.00 & 18.91 & 67.75 & 19.36 & 71.27 & 16.62 & 72.17 & 16.76 & 2.01 & .111 \\
\hline Auto-eficacia modelo textual & 70.80 & 15.48 & 70.18 & 14.00 & 74.03 & 14.32 & 73.14 & 14.64 & 2.19 & .088 \\
\hline $\begin{array}{l}\text { Auto-eficacia modelo } \\
\text { decodificación y fluidez }\end{array}$ & 75.06 & 21.18 & 75.93 & 18.02 & 81.44 & 14.31 & 79.02 & 15.89 & 3.65 & .012 \\
\hline
\end{tabular}

Por su parte los análisis post hoc mostraron en la auto-eficacia en relación a los procesos de bajo nivel, decodificación y fluidez, diferencias estadísticamente 
significativas entre los cursos de $1^{\circ}$ y $3^{\circ}$ de Educación Secundaria obligatoria, con $p=.02$, reflejándose un incremento del nivel de auto-eficacia en esta dimensión con el curso, tal como se puede observar en los estadísticos descriptivos de la tabla 2. Por su parte, los análisis post hoc entre los grupos para la auto-eficacia total no alcanzaron una significatividad estadística entre los pares de grupos. No obstante, nuevamente la tendencia general observada entre los cursos es la de un incremento en el nivel de autoeficacia total con la edad.

\section{DISCUSIÓN Y CONCLUSIONES}

A partir de los resultados obtenidos cabe sugerir la existencia de diferencias estadísticamente significativas en el patrón de desarrollo de la auto-eficacia lectora entre los cursos de $1^{\circ}$ a $4^{\circ}$ de Educación Secundaria Obligatoria, en relación a las medidas de auto-eficacia total y auto-eficacia en relación a los procesos de decodificación y fluidez, mostrándose en general un aumento del nivel de auto-eficacia lectora a medida que aumentan los cursos.

Este patrón evolutivo es coherente con los resultados obtenidos en estudios previos como el citado anteriormente de Mucherah y Yoder (2008) si bien dicho estudio se realizó con una muestra de 388 estudiantes de 11 y 13 años, pertenecientes al primer ciclo de Educación Secundaria.

Una posible explicación a estos resultados se puede encontrar en que los alumnos de cursos superiores como consecuencia de un dominio y automatización de los procesos de bajo nivel cognitivo implicados en la lectura, han logrado unas creencias de competencia en relación a estos procesos significativamente mayor que los alumnos de los cursos iniciales, en los que proviniendo de la Educación Primaria, todavía pueden estar habituados aún a un mayor énfasis en aspectos mecánicos de fluidez lectora.

Sin embargo, en relación a los procesos de alto nivel cognitivo, aquellos realmente claves para un óptimo rendimiento lector, no hemos encontrado la misma tendencia. Así, los alumnos de cursos superiores no se sienten más capaces que los alumnos de los cursos inferiores, tal y como cabría esperar. Resultado que puede tener implicaciones muy negativas en relación al logro de la competencia lectora del alumnado. Dichos resultados podrían encontrar su explicación en que los alumnos de cursos inferiores están sobreestimando sus habilidades lectoras, mostrando unas creencias de competencia positivas muy elevadas lo cual, si no va unido a un dominio real a nivel estratégico y autorregulado de lectura, puede llevar precisamente al efecto contrario al esperado por un nivel de auto-eficacia lectora alto, tal y como defiende Bandura (1997). Este hecho podría incidir en los problemas en comprensión lectora que se han reflejado en los correspondientes informes Pisa que presenta el alumnado de primer ciclo de Secundaria (segundo curso). 
Por lo tanto, el primer ciclo de Educación Secundaria Obligatoria parece un periodo crítico a considerar a nivel de intervención en comprensión lectora. Las creencias de auto-eficacia lectora del alumnado en este periodo son altas, lo que tal como se ha recogido anteriormente, influye positivamente a nivel conductual, motivacional y cognitivo en el aprendizaje; es por ello clave el dotarles en ese momento del dominio auto-regulado y metacognitivo de diferentes estrategias de lectura, dirigidas a los procesos de alto nivel cognitivo, que permitan a la auto-eficacia generar un patrón adaptativo, y positivo para el aprendizaje. A su vez, el dotar al alumnado en este ciclo inicial de la secundaria de las estrategias y de las creencias motivacionales óptimas para la aplicación de dichas estrategias lectoras, permitirá afrontar los cursos superiores, en los que las demandas lectoras aumentan significativamente, de una manera óptima por el alumnado.

No obstante, dichas conclusiones deben considerarse dentro de las limitaciones del presente estudio, ya que si bien hemos analizados los cambios en el nivel de auto-eficacia lectora a través de los cursos escolares, no se han tenido en cuenta los cambios en su calibración, aspecto clave para poder determinar con mayor certeza las diferencias reales entre los cursos, la mayor adaptación o no de sus creencias motivacionales, y su incidencia positiva o negativa en la competencia lectora del alumnado, y por ende, en su aprendizaje; puesto que parafraseando a Bandura, el nivel auto-eficacia óptimo para el aprendizaje es aquél que sobre-estima ligeramente el nivel real de competencia de la persona. Todo ello, abre una nueva línea de estudio que se está desarrollando en posteriores investigaciones.

Agradecimientos: Esta investigación forma parte del proyecto de investigación con Referencia EDU2010-18219, concedido por el Ministerio de Ciencia e Innovación a la Dra. Fidalgo.

\section{REFERENCIAS}

Bandura, A. (1997). Self-efficacy: The Exercise of Control. New York: Freeman.

Bandura, A. (2006). Guide for constructing self-efficacy scales. En F. Pajares, y T. Urdan (Eds.), Self-efficacy beliefs of adolescents (pp. 307-337). Greenwich, Connecticut: Information Age Publising.

Fidalgo, R., Arias-Gundín, O. y Olivares, F. (2013). Cuestionario de Auto-eficacia en la Comprensión lectora. Aula Abierta, 41(1), 17-26.

Guthrie, J.G. y Wigfield, A (2000). Engagement and motivation in reading. En M.L. Kamil, P.B. Mobenthal, P.D. Pearson y R. Barr (Eds.), Handbook of reading research, 3, 403-420.

Kelley. J. y Decker, E.O. (2009). The current state of motivation to read among middle school students. Reading Psychology, 30, 466-485.

Kintsch, W. y Rawson, K. (2005). Comprehension. En M. Snowling, y C. Hulme. The science of reading. A handbook (pp. 209-226). Oxford: Blackwell. 
Lau, K. (2009a). Grade differences in reading motivation among Hong Kong primary and secondary students. British Journal of Educational Psychology, 79, 713-733.

Lau, K. (2009b). Reading motivation, perceptions of reading instruction and reading amount: a comparison of junior and senior secondary students in Hong Kong. Journal of Research in Reading, 32, 366-382.

Linnenbrink, E.A. y Pintrich, P.R. (2003). The role of self-efficacy beliefs in student engagement and learning in the classroom. Reading and Writing Quarterly, 19, 119-137.

Mattus, J.A., Pérez, E., Pujol, M.A. y Casasola, I.A. (2011, Marzo). Desarrollo y validación preliminar de una escala de auto-eficacia para la comprensión lectora. Comunicación presentada en el VI Congreso Internacional de Psicología y Educación de la Asociación Nacional de Psicología y Educación en España, Valladolid.

Mucherah, W. y Yoder, A. (2008). Motivation for Reading and middle school students' performance on standardized testing in reading. Reading Psychology, 29, 214-235.

Nelson, J.N. y Manset-Williamson, G. (2006). The impact of explicit, self-regulatory reading comprehension strategy instruction on the reading-specific self-efficacy, attributions, and affect of students with reading disabilities. Learning Disability Quarterly, 29, 213230.

OECD (2009). PISA 2009 Assessment Framework. Paris: OECD.

Olivares, F. y Fidalgo, R. (2012). El estado de la Cuestión sobre el Estudio de la Auto-eficacia Lectora en Alumnado con y sin Dificultades de aprendizaje. Aceptado para publicación.

Pajares, F. (2003). Self-efficacy beliefs, motivation, and achievement in writing: A review of the literature. Reading y Writing Quarterly, 19, 159-172.

Pajares, F. (2003). Self-efficacy beliefs, motivation, and achievement in writing: A review of the literature. Reading y Writing Quarterly, 19, 159-172.

Vidal-Abarca, E., Gilabert, R., Martínez, T., Sellés, P., Abad, N. y Ferrer. (2007). Test de Estrategias de Comprensión, TEC. Madrid: ICCE.

Wigfield, A., Guthrie, J.T., Tonks, S. y Perencevich, K.C. (2004). Children's motivation for reading: Domain specificity and instructional influences. Journal of Educational Research, 97(6), 299-309.

Zimmerman, B.J. (2000). Self-Efficacy: An Essential Motive to Learn. Contemporary Educational Psychology 25, 82-91. doi:10.1006/ceps.1999.1016.

Recibido: 2 de marzo de 2013 Recepción Modificaciones: 8 de marzo de 2013 Aceptado: 23 de agosto de 2013 